\title{
NUEVOS ELEMENTOS ORIENTALIZANTES EN LA COMARCA DE GILENA
}

\section{NEW ORIENTALISING ELEMENTS IN THE GILENA SECTOR (SEVILLA)}

\author{
por \\ JULIÁN MANCEBO DÁVALOS
}

RESUMEN De la comarca de Gilena, contamos con algunos trabajos referentes a su poblamiento en época calcolítica, con yacimientos como El Negrón, y otros estudios de prospección que han permitido documentar otros horizontes culturales protohistóricos, con asentamientos como Cerro Gordo, del que ya se han publicado sus cerámicas pintadas orientalizantes e ibéricas, o Los Villares, del que también se conocían materiales de ese momento.

Los nuevos elementos que analizaremos en este trabajo, cerámicas grises y puntas de flechas, amplían el conocimiento del fenómeno orientalizante en la campiña y sierra suroriental de la provincia de Sevilla.

ABSTRACT From the Gilena area we have some items belonging to its calcolothic settlement with sites like the one called "El Negrón". There are also some prospect studies that have brought information about other protohistoric culturals horizons with sites such as those known as "Cerro Gordo" and "Los Villares". From the first one we know its orientalizing and iberian painted pottery which has already been published, and from the second one, many objetcs were also known.

The new items to be analized here, that is, grey pottery and arrow edges, enlarge our knowledge about the Orientalizing Period in the South-East area in Sevilla province.

El Término de Gilena se ubica en el extremo suroriental de la Provincia de Sevilla, y a ella, ya se refieren los autores del s. XIX como Madoz, citando entre sus restos arquitectónicos el castillo de Ipora (1.847: 420), en cuyas cercanías se localizó el yacimiento de Cerro Gordo. Posteriormente, es mencionado su estudio en el Catálogo Arqueológico de la Provincia, donde se sitúan puntos como la Loma de las Flores (780 m.), o la de Acebuchosa (660 m.), y puntos de menores alturas como Espartales (Hernández y otros, 1.955: 183).

En los últimos años se ha centrado el interés por el estudio del poblamiento calcolítico, con actuaciones en yacimientos como El Negrón, donde se utilizan métodos geofísicos (Cruz-Auñón y Rivero, 1.986), o sobre la época romana (Larrey y Morales, 1.986), lo que motiva la realización de Cartas Arqueológicas, y las prospec- 
ciones llevadas a cabo en la comarca de Osuna, y en el Término de Gilena (Romo y otros, 1.990; Pérez y otros, 1.989) ${ }^{1}$, por lo que en este artículo, complementaremos estos estudios con nuevos elementos orientalizantes.

- Cerro Gordo (Gilena): Situado en un cerro de escasa altitud $(370 \mathrm{~m}$.), pertene a las primeras estribaciones de la Sierra de Estepa (Fig. 1).

Conocemos de este yacimiento la descripción de ciertos materiales que reflejan un poblamiento en el Bronce Final, con cerámicas a mano propias de este momento, y de la edificación de una estructura fortificada de difícil adscripción cronológica (Romo y otros, 1.990: 311).

Del período orientalizante, que aquí nos interesa, conocemos la publicación de sus cerámicas pintadas a torno, entre las que destacan algunas producciones de barniz rojo, y las cerámicas con motivos figurativos, donde se identifican representaciones florales y zoomorfos (Bandera y otros, 1.989). Durante los trabajos de prospección, también se documentaron un número de piezas correspondientes a cerámicas grises que describiremos a continuación:

1. Borde de cuenco de perfil hemisférico o troncocónico (Fig. 2.2), tipos I/II de Belén para los ejemplares de Huelva (1.976: 367), 20.A de Caro (1.986: 734), o I.A de Mancebo para el Bajo Guadalquivir (1.994: 352). Son característicos en la producción cerámica gris, y se difunden tanto en yacimientos coloniales, como en el núcleo tartésico y su hinterland, en ambientes cronológicos de los s. VII y VI a.C.

2. Doce fragmentos de cuencos hemisféricos con bordes compuestos y engrosados (Fig. 2.1), tipos 20.B de Caro (1.986: 734), o I.C de Mancebo (1.994: 353), siendo formas características de los s. VII-VI a.C. en los asentamientos orientalizantes del Bajo Guadalquivir.

3. Fragmento de plato con carena media y borde convexo ancho (Fig. 3.1). Responde al tipo V/VI de Belén (1.976: 370), 17.B de Caro (1.986: 689), o II.D.1 de Mancebo (1.994: 356), y surgen en la Península a partir de modelos realizados en barniz rojo (Schubart y otros, 1.969: Lám. X), documentándose en la Cuenca del Guadalquivir desde el s. VIII a.C. (Mancebo, 1.994: 356).

4. Fragmento de plato de perfil compuesto (Fig. 3.2), tipo 11 de Caro (1.986: 619), y II.F de Mancebo (1.994: 357). Se documenta en algunas prospecciones de la Vega del Corbones, y en Montemolín, desde los inicios del Orientalizante en el s. VII hasta el s. V a.C, y los niveles del saqueo que sufrió en época ibérica su sector oriental (Mancebo y otros, 1.992: 287).

5. Fragmento de cuenco carenado (Fig. 2.3), tipo III de Mancebo (1.994: 358), y B de Lorrio (1.988-89: 296), que se documentan en los niveles antiguos de los yacimientos orientalizantes, imitando formas cerámicas a mano del Bronce Final desde fines del s. VIII a.C. (Mancebo, 1.994: 358).

6. Dos fragmentos de vasos cerrados globulares con carena alta y cuello corto (Fig. 3.3), tipos 5 de Caro (1.986: 558), o VI de Mancebo (1.994: 360), de tradición indígena y documentado en el Bajo Guadalquivir entre los s. VII y VI a.C.

7. Fragmento de olla o urna de forma ovoide o globular (Fig. 2.4), tipo 7 de Caro (1.986: 577), formas D.1A/7C de Lorrio (1.988-89: 300), y VIII de Mancebo (1.994: 360), con ejemplos en el s. VII a.C., aunque son frecuentes en ambientes del s. VI a.C. en poblados como Montemolín (Mancebo y otros, 1.992: 289), o los N. 20 y 18 del Cerro Macareno (Pellicer y otros, 1.983: fig. 56).

8. Fragmento de borde exvasado de una vasija globular tipo "urna Cruz del Negro" (Fig. 3.4). Forma 8 de Caro (1.986: 591), D.2/6 de Lorrio (1.988-89: 300), o X de Mancebo (1.994: 361), propios de niveles del s. VI a.C. en los yacimientos del Sur hispano.

1. Desde estas líneas queremos expresar nuestra gratitud a los autores de estas prospecciones, que nos han dejado estudiar sus materiales inéditos y nos han brindado toda la información disponible sobre los yacimientos referidos. 
9. Diez fragmentos de fondos rehundidos y marcados (Fig. 3.5), tipo B de Montemolín (Mancebo, 1994b:106), entre los que destaca un ejemplar en el cual el pie desarrolla casi un solero (Fig. 3.6).

10. Dos fragmentos de fondos planos con pies marcados (Fig. 3.7), tipo D de Montemolín (Mancebo, 1994b: fig. 2), donde se fechan en niveles del s. VI a.C. Son piezas que deben pertenecer a vasos de forma abierta, ya que estadísticamente son las más frecuentes en este área, como atestiguan los ejemplares de Cerro Gordo.

Las cerámicas grises de este asentamiento responden, como es norma común en el resto de yacimientos analizados en el Bajo Guadalquivir, a formas abiertas de platos y cuencos, con escasa representación de ejemplares cerrados -algún fragmento de olla-, ya que en estos poblados protohistóricos, los recipientes de cocina, continuarán fabricándose en cerámicas a mano (Mancebo y otros, 1.992: 290).

De los datos que nos ofrece el análisis comparativo con yacimientos de su entorno, situamos en el s. VI a.C el momento de mayor desarrollo de esta vajilla en el poblado, con formas características de estos momentos como los cuencos de borde engrosado. Fecha atestiguada también por otras producciones cerámicas de $\mathrm{C}$. Gordo, como sus ánforas de transporte, con perfiles protoibéricos, y los recipientes de barniz rojo o cerámicas pintadas, entre las que los motivos figurativos, zoomorfos y fitomorfos destacan especialmente, con temas bien documentados dentro de esta centuria (Pérez y otros, 1.989: Fig. 3; Bandera y otros, 1.989: 304).

- Los Villares (Gilena): (Fig. 1) Cerro situado al pie de la vía romana que atraviesa el Término Municipal de Gilena cruzando la Cañada Real de Granada (Hernández y otros, 1.955: 191).

Catalogado como yacimiento turdetano en los trabajos arqueológicos publicados sobre la zona, por algunos fragmentos de cerámica pintada, otra pieza con motivo floral nos recuerda a la cerámica figurativa orientalizante comentada de C. Gordo (ibid, 1.955: 185; Romo y otros, 1.990: 311).

Este yacimiento ofrece, fruto de las prospecciones clandestinas efectuadas con los célebres detectores de metales, un lote de puntas de flecha de bronce (Ferrer, 1993), propias del período orientalizante, datadas en los s. VII y VI a.C. (Mancebo y Ferrer, 1.988-89: 328), y que responde básicamente a dos tipos de la clasificación de Ramón (1.983), con nueve ejemplares de su tipo 11.A, de hoja lanceolada de doble filo, y arpón lateral. Y una pieza de su tipo 31, con hoja triangular de doble filo y la base cóncava, que hace innecesaria la presencia del arpón (Fig. 4, 8).

En cuanto al origen de este grupo de puntas de flecha, consideramos que no es preciso referir de nuevo las argumentaciones expuestas por otros investigadores en favor del origen oriental de estos artefactos, centrado en la zona del Mar Negro (García Guinea, 1.967, 95), que nosotros aceptamos y hemos defendido anteriormente (Mancebo y Ferrer, 1.988-89, 326).

Todas las puntas de este yacimiento, presentan señales de su utilización como flechas, algunas con sus extremos rotos o desgastados, y algunos orificios practicados para fijar un remache en el cubo de la pieza, donde apreciamos el cañón roto por el impacto de la punta sobre una superficie dura, como sería el lienzo de muralla donde se han recuperado en Pancorvo, hecho frecuente en los artefactos documentados en la zona (Fig. 4), como las piezas recogidas en el foso de Acinipo (comunicación oral de sus excavadores), o formando parte de estratos de incendio y destrucción en Peña Negra o Macalón. De ahí su estado de traumatismo, como se las documenta en estos asentamientos (Mancebo y Ferrer, 1.988-89, 316). Hecho que también está atestiguado en otros puntos extrapeninsulares como Kamir-Blou en Armenia, en niveles de destrucción de la ciudad, o el ejemplar aparecido en la fortaleza de Salmansar, que se relaciona con el asedio del año 612 a.C. (García Guinea, 1.967, 81-84). Por lo tanto, habría que relacionar estas piezas con una serie de conflictos armados que se desarrollarían principalmente a partir del s. VI a.C., y cuyo registro estratigráfico está ampliamente constatado en los yacimientos orientalizantes (Ferrer, 1993).

Al analizar los ejemplares de esta comarca, interesa también destacar el circuito de penetración de estos artefactos a partir de las rutas gaditanas y malagueñas, que ponen en contacto la vertiente mediterránea con las 
campiñas sevillanas y cordobesas, donde se plantea también la posibilidad de contar con algún taller local debido a la gran concentración de estos ejemplares en esta zona (Quesada, 1.988).

Estos yacimientos de C. Gordo y Los Villares, se presentan pues como enclaves de primer orden, con elementos materiales que nos sitúan plenamente dentro del s. VI a.C, por los análisis comparativos efectuados con otros poblados protohistóricos del entorno, con cerámicas grises, pintadas figurativas, puntas de flechas, o estructuras defensivas, que incluyen a estos asentamientos en la dinámica orientalizante del Sur Peninsular, y ubicados en una zona excelente como eje de comunicaciones entre la vertiente malagueña, donde se sitúan preferentemente los yacimientos coloniales semitas, y la Campiña y Vega del Guadalquivir, área nuclear del fenómeno tartéssico andaluz.

\section{BIBLIOGRAFÍA}

BANDERA, M.L. y otros (1989): "Cerro Gordo, un yacimiento Orientalizante de la Sierra Sur Sevillana (Gilena, Sevilla)", Habis, 20: 293-306.

BELÉN DEAMOS, M․ (1976): "Estudio y tipología de la cerámica gris en la provincia de Huelva", Revista de Archivos, Bibliotecas y Museos LXXIX, 2: 353-388, Madrid.

CARO, A. (1986): Las cerámicas grises a torno orientalizantes de Andalucía, Tesis Doctoral, Cádiz.

CRUZ-AUÑÓN, R. y RIVERO, E. (1986): "Yacimiento del Negrón. Cueva Antoniniana", Excavaciones arqueológicas. Gilena.

FERRER, E. (1993): Nuevos documentos arqueológicos para la definición del horizonte orientalizante en la Península Ibérica: las puntas de flecha, Tesis de Licenciatura inédita.

GARCÍA GUINEA, M.A. (1967): "Las puntas de flecha con anzuelo y doble filo y su proyección hacia Occidente", A.E.A. 40: 69-87.

HERNÁNDEZ DÍAZ, J. y otros (1955): Catálogo Arqueológico y Artístico de la provincia de Sevilla, IV, Sevilla.

LARREY, E. y MORALES, M.I. (1986): "Investigación arqueológica en las termas romanas de Aparicio el Grande (Gilena)", Excavaciones arqueológicas. Gilena

LORRIO ALVARADO, A. (1988-89): "Cerámica gris orientalizante de la necrópolis de Medellín (Badajoz)", Zephyrus LI-LII: 283-314.

MADOZ, P. (1847): Diccionario geográfico-estadístico de España y posesiones de ultramar, VIII, Madrid.

MANCEBO, J. y FERRER, E. (1988-89): "Aproximación a la Problemática de las Puntas de Flecha en el Período Orientalizante. El Yacimiento de Pancorvo (Montellano, Sevilla). Zephyrus XLI-XLII: 315-330.

MANCEBO, J. y otros (1992): "La cerámica gris a torno del yacimiento orientalizante de Montemolín (Sevilla)", T.P, 49.

MANCEBO, J. (1994): "Las cerámicas grises a torno orientalizantes de la Cuenca Baja del Guadalquivir", Arqueología en el entorno del Bajo Guadiana: 351-373.

-(1994b): "Consideraciones sobre la cerámica gris a torno de Montemolín (Sevilla)",Zephyrus, XLVII:105111.

PELLICER, M. y otros (1983): El Cerro Macareno, E.A.E. 124.

PÉREZ, J.A. y otros (1989): "Asentamientos ibero-turdetanos en el extremo Suroriental de la Campiña Sevillana (Comarca de Osuna)", Estudios sobre Urso: 187-211, Sevilla.

QUESADA SANZ, F. (1988): “Nuevas puntas de flecha de anzuelo en Andalucía Occidental”, Ariadna, 5: 1-15. RAMÓN, J. (1983): " Puntas de flecha de bronce fenicio-púnicas halladas en Ibiza: algunos materiales inéditos", Homenaje al Profesor M. Almagro Basch, II: 309-323, Madrid. 
ROMO, A. y otros (1990): "Prospección arqueológica superficial del T.M. de Gilena (Sevilla)", A.A.A.' $88 / I I I$ SCHUBART, H. y otros (1.969): Toscanos. La factoría paleopúnica en la desembocadura del río Vélez, E.A.E. 66.

ULREICH, H. y otros (1990): "Cerro del Prado. Die Ausgrabungen 1989 im Schuttang der phönizischen Ansiedlung an der Guadarranque-Mündung”, M.M., 31: 194-250. 


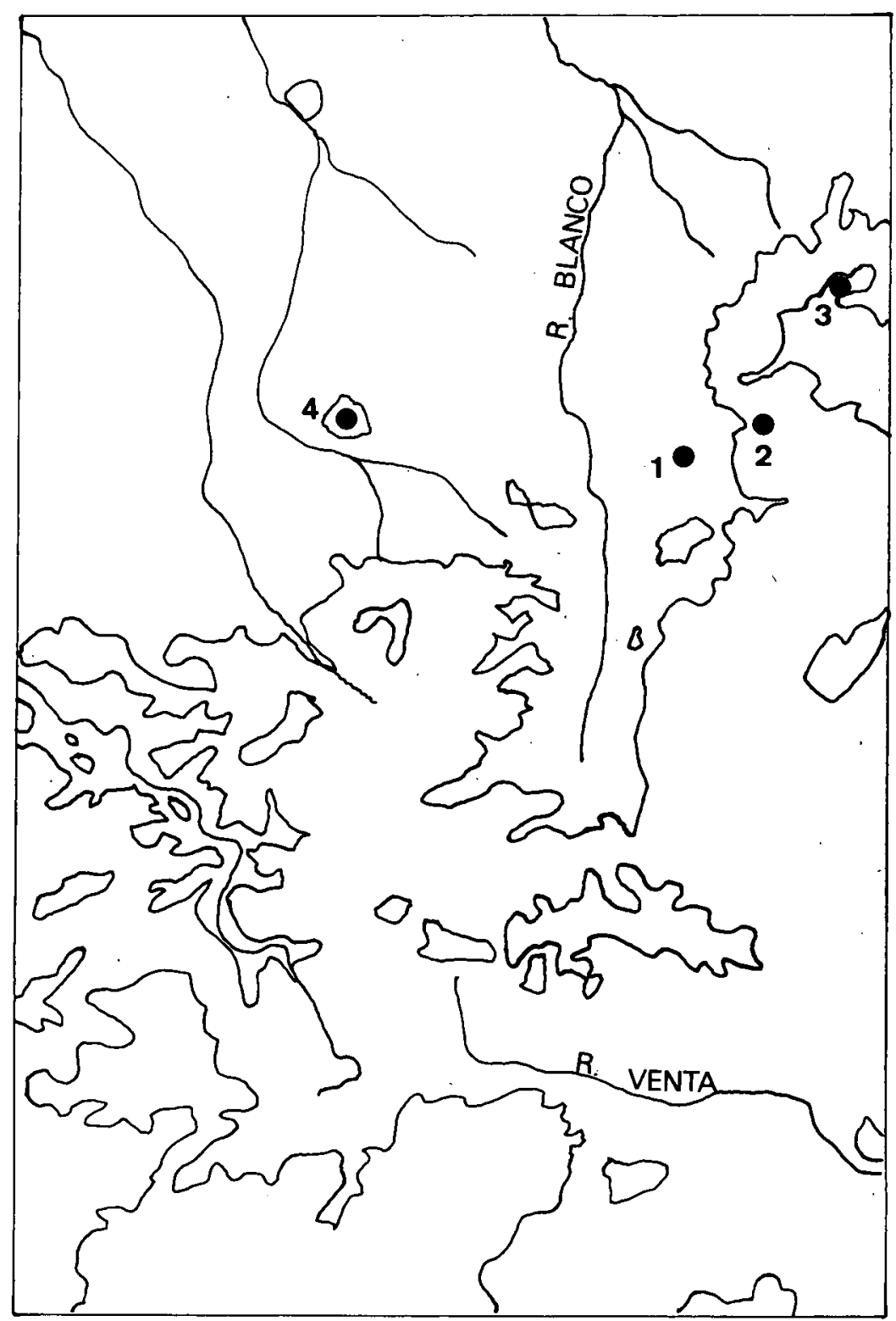

Fig. 1. Mapa de localización de yacimientos: 1) C. GORDO (GILENA). 2) LOS VILLARES (GILENA). 3) ESTEPA. 4) OSUNA. 


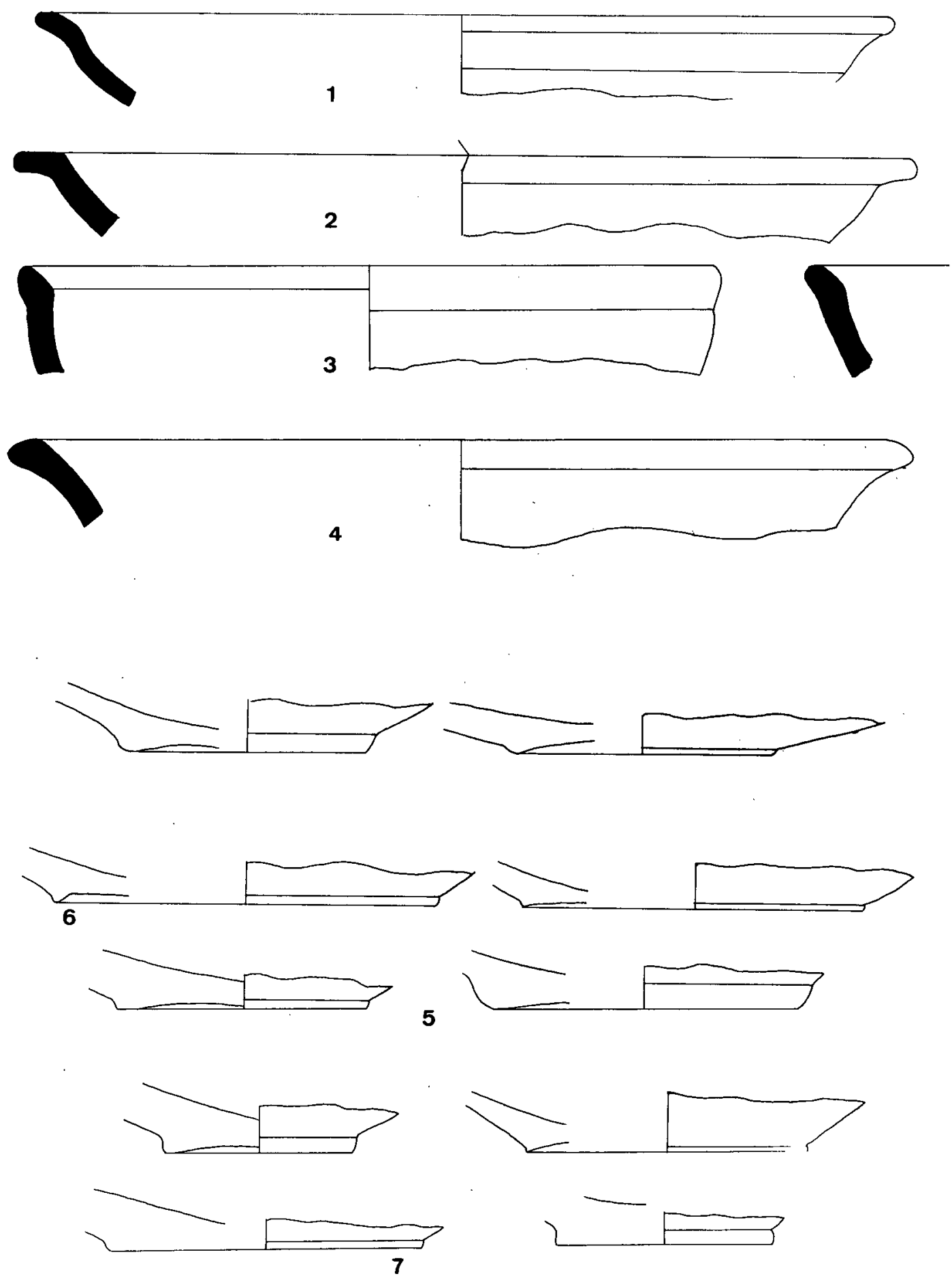

Fig. 3. Cerámicas grises de C. Gordo (Gilena). 

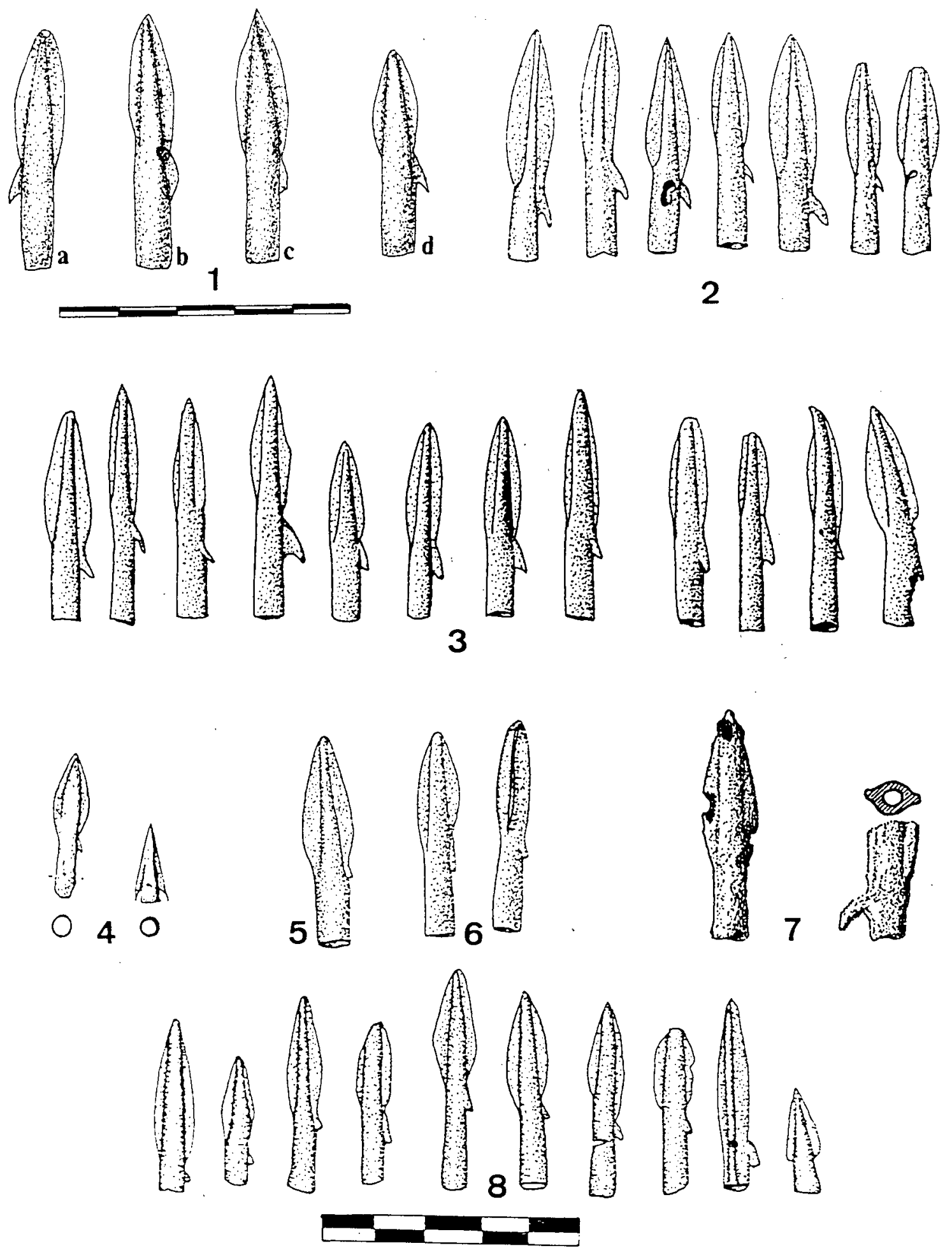

Fig. 4. Algunos ejemplos de puntas de flecha orientalizantes: 1) puntas del Pantano del Chorro (Mancebo 1.994 e.p); 2) Otras puntas del Pantano del Chorro (Ferrer, 1.993); 3) El Hacho de Benamejí (Ferrer, 1.993); 4) C. del Prado (Ulreich y otros, 1.990); 5) Montemolín (Ferrer, 1.993); 6) C. de la Atalaya en Villanueva de S. Juan (Ferrer, 1.993); 7) Toscanos (Schubart y Niemeyer, 1.969); 8) Los Villares de Gilena (Ferrer, 1.993). 